In addition, DNA damage and p53 pathway genes were upregulated upon $A L K B H 1$ silencing in hypoxia. Together, these findings imply that ALKBH1 changes the $N^{6}-\mathrm{mA}$ landscape to control key oncogenic pathways during glioblastoma progression.

Hoping to identify $N^{6}-\mathrm{mA}$ as a therapeutic vulnerability, the authors found that ALKBH1 knockdown reduced both GSC proliferation and sphere formation, a surrogate marker for self-renewal. Furthermore, mice bearing autochthonous tumours formed from GSC lines expressing shRNAs targeting ALKBH1 exhibited extended survival over mice implanted with GSC lines expressing control shRNAs.

In the future, it will be interesting to determine how DNA $N^{6}-\mathrm{mA}$ and H3K9me3 cooperate with other DNA modifications, such as $5 \mathrm{mC}$, to coordinate transcriptional silencing in human cancers.

Anna Dart

ORIGINAL ARTICLE Xie, Q., Wu, T.P. E Gimple, R. C. et al. N ${ }^{6}$-methyladenine DNA modification in glioblastoma. Cell https://doi.org/ 10.1016/j.cell.2018.10.006 (2018)

the changes in the TCR repertoire had the highest predictive value of response.

Examination of the tumour specificity of the expanded T cell clones in one patient with a complete response indicated that this patient had tumour-infiltrating lymphocytes that matched the expanded clones. The authors also identified two expanded $\mathrm{T}$ cell clones in the periphery in this patient, both of which recognized a neoantigen derived from a protein, karyopherin $\alpha 2$, thought to be upregulated during radiotherapy.

Thus, the authors hypothesize that radiotherapy may increase expression of immunogenic mutations in the tumour, leading to activation of T cells that induce IFN $\beta$, suggesting a possible future role for combination therapy with ipilimumab and radiotherapy.

Jordan Hindson

ORIGINAL ARTICLE Formenti, S. C. et al. Radiotherapy induces responses of lung cance to CTLA-4 blockade. Nat. Med. 24, 1845-185 (2018)

FURTHER READING Ngwa, W. et al. Using immunotherapy to boost the abscopal effect. Nat.Rev. Cancer 18, 313-322 (2018)

\title{
Dividing paths in fatty liver disease
}

Nonalcoholic steatohepatitis (NASH) is associated with obesity and is a more severe and progressive form of nonalcoholic fatty liver disease (NAFLD), showing advanced fibrosis and hepatic inflammation. Simple steatosis is a less severe form of NAFDL and can progress into NASH. A subset of patients with NASH develop hepatocellular carcinoma (HCC) with advanced fibrosis and cirrhosis, while others do not. Also, an increasing number of patients with simple steatosis develop HCC but without symptoms of advanced fibrosis and cirrhosis, indicating that NASH might not be required for HCC development. Grohmann, Wiede et al. have now reported in Cell that the pathologies of NASH and $\mathrm{HCC}$ in obesity can be driven by two independent pathways.

Oxidation of protein tyrosine phosphatases (PTPs), which leads to their inactivation, occurs in livers of high-fat diet (HFD)-fed mice that develop NAFL but not NASH. Following up on their previous studies and looking into whether PTP oxidation is associated with NASH and/or HCC, the researchers used C57BL/6 mice fed with a normal chow diet (NCD), HFD (promoting obesity, insulin resistance and simple steatosis) or choline-deficient HFD (CD-HFD; promoting obesity, insulin resistance and progression of simple steatosis to NASH). Indeed, PTP oxidation status, including that of T cell PTP (TCPTP), was increased in HFD-fed mice and further increased in CD-HFD fed mice, compared with NCD mice.

The researchers chose to focus on TCPTP, because TCPTP oxidation was higher in livers of obese patients with NAFLD compared with

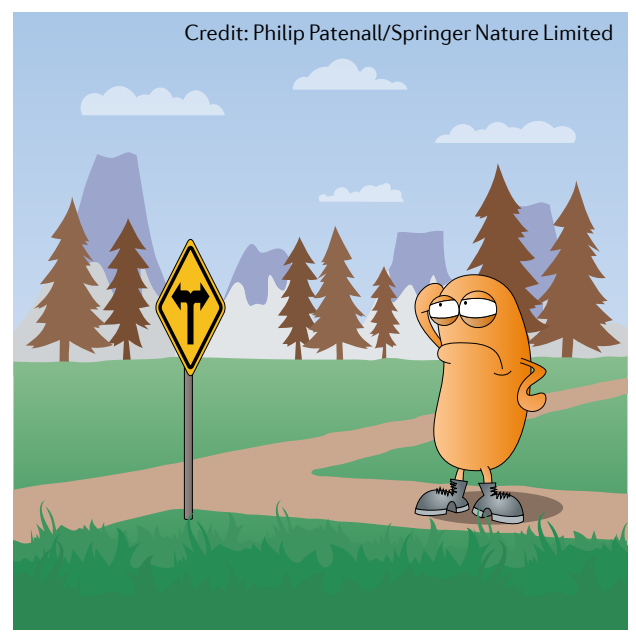

livers of those without steatosis. TCPTP dephosphorylates and inactivates STAT1 and STAT3, and phosphorylation of STAT1 and STAT3 was increased in livers of HFD and CD-HFD mice compared with NCD mice as well as in livers of obese patients with NAFLD compared with non-obese patients without NAFLD. C57BL/6 mice with deletion of TCPTP in hepatocytes (Alb-Cre;Ptpn2 $2^{f / f l}$ ) but not control mice (Ptpn $\left.2^{f / f l}\right)$ developed NASH after being fed a HFD, which included NASH-associated increased immune cell infiltration and ectopic lymphoid-like structure formation, as well as increased liver damage. Immune cell infiltrates were composed of increased numbers of $\mathrm{CD}^{+}$and $\mathrm{CD} 8^{+} \mathrm{T}$ cells, and also contained increased numbers of immunoglobulin $A$ $(\lg \mathrm{A})$-positive cells, which express high levels of programmed cell death 1 ligand 1 (PD-L1) compared with Ptpn $2^{f / f l}$ mice. Importantly, one in three Alb-Cre;Ptpn $2^{f l / f l}$ mice fed with HFD developed aggressive HCC, in contrast to HFD-fed Ptpn $2^{f l / f l}$ mice, none of which developed HCC.

To analyse STAT1 and/or STAT3 function in $\mathrm{NASH}$ and HCC development, the researchers generated Alb-Cre;Ptpn2 $2^{\text {fl/fl }}$ mice with heterozygous deficiency of STAT1 or STAT3, leading to correction of the increased phosphorylation of either protein in hepatocytes. Importantly, STAT1 heterozygosity $\left(\right.$ Stat $\left.1^{f /+}\right)$ repressed hepatic inflammation, decreased immune cell recruitment and decreased fibrosis in HFD-fed Alb-Cre;Ptpn $2^{f / f l}$ mice, compared with control mice. By contrast, STAT3 heterozygosity (Stat $3^{f / /+}$ ) did not reduce immune cell recruitment or fibrosis in HFD-fed Alb-Cre;Ptpn $2^{f l / f l}$ mice, compared with control mice. However, again in contrast to STAT1 heterozygosity, STAT3 heterozygosity completely repressed the development of HCC in HFD-fed Alb-Cre;Ptpn $2^{f / f l}$ mice, compared with control mice.

$\mathrm{NASH}$ and HCC in obesity are driven by increased STAT1 and STAT3 signalling, respectively, as a result of increased TCPTP oxidation. Although requiring validation, this study calls into question the predominant view of NASH preceding $\mathrm{HCC}$ in obesity.

Ulrike Harjes

ORIGINAL ARTICLE Grohmann, M., Wiede, F. et al. Obesity drives STAT-1-dependent NASH and STAT-3-dependent HCC. Cell https://doi.org/10.1016/j.cell.2018.09.053 (2018) 